\title{
Autologous stem cell transplantation in a patient with systemic sclerosis-associated interstitial lung disease in Taiwan
}

\author{
Yu-Jen Pan, ${ }^{1,4}$ Wei-Han Huang, ${ }^{2,3,4}$ Kuei-Ying Su ${ }^{1,4}$
}

\begin{abstract}
Background: Systemic sclerosis (SSc), characterized by fibrosis of the skin and other organs, is a devastating systemic autoimmune disease. Lung involvement is frequent in SSc and contributes to the high rate of mortality.

Objective: To describe the first case report of SSc and interstitial lung disease (ILD) receiving autologous hematopoietic stem cell transplantation (ASCT) in Taiwan.

Methods: Case report.

Results: A 51-year-old man presented with rapid skin thickening and shortness of breath. Early progressive SSc-associated ILD was diagnosed. Because his lung function rapidly declined and his skin disease progressed, he received ASCT with satisfactory treatment responses in both skin thickness and lung function. In addition, lung imaging analysis showed remarkable improvements in ILD after treatment.
\end{abstract}

Conclusion: We suggest that ASCT can be considered in selected patients with early, rapidly progressive SSc associated with ILD.

Key words: systemic sclerosis, scleroderma, therapy, adult stem cells, autologous transplantation, interstitial lung disease.

From:

${ }^{1}$ Division of Allergy, Immunology \& Rheumatology,

Hualien Tzu Chi Hospital, Buddhist Tzu Chi Medical Foundation, Hualien, Taiwan

${ }^{2}$ Department of Clinical Pathology, Hualien Tzu Chi Hospital, Buddhist Tzu Chi Medical Foundation, Hualien, Taiwan

${ }^{3}$ Division of Oncology and Hematology, Hualien Tzu Chi Hospital, Buddhist Tzu Chi Medical Foundation, Hualien, Taiwan

${ }^{4}$ School of Medicine, Tzu Chi University, Hualien, Taiwan

\section{Introduction}

Systemic sclerosis (SSc) is a rare autoimmune disease characterized by progressive fibrosis of the skin and internal organs. Interstitial lung disease (ILD), a common extracutaneous manifestation, is one of the leading causes of death in patients with SSc. In the European League Against Rheumatism (EULAR) Scleroderma Trials and Research cohort, 55\% of deaths among SSc patients were directly related to SSc, and $35 \%$ by pulmonary fibrosis. ${ }^{1}$ In a retrospective cohort, the extent of lung involvement on high-resolution computed tomography and low forced vital capacity were associated with higher mortality rates. ${ }^{2}$ Many patients with SSc-ILD

\section{Corresponding author:}

Kuei-Ying Su

Division of Allergy, Immunology and Rheumatology,

Hualien Tzu Chi Hospital, Buddhist Tzu Chi Medical Foundation,

No. 707, Sec. 3, Chung-Yang Rd., Hualien City, Hualien County 970,

Taiwan

E-mail: kueiying.su@tzuchi.com.tw

have traditionally been treated with immunosuppressants, such as cyclophosphamide and mycophenolate; however, these drugs have only limited effects than placebo. ${ }^{3}$ The difference in forced vital capacity percent predicted at 12-month was only $2.53 \%$ between cyclophosphamide and placebo. ${ }^{4}$ In addition, there is no evidence of survival benefit from adding immunosuppressants in these trials. ${ }^{3}$

Since 2016, the EULAR has recommended autologous hematopoietic stem cell transplantation (ASCT) for selected patients with rapidly progressive SSc who are at risk for organ failure, ${ }^{5}$ this recommendation was based upon the favorable 
results from clinical trials with selected patients, and has been further supported by safety reports from long-term follow-up studies. ${ }^{6,7,8}$

Medical centers in Taiwan have extensive experience with the use of ASCT in patients with various hematologic diseases. ${ }^{9}$ However, ASCT treatment for patients with SSc-ILD has not been reported in Taiwan. We describe a patient in whom SSc-ILD was successfully managed with non-myeloablative ASCT, with significant improvements in both skin and lung diseases.

\section{Case report}

A 51-year-old man was presented with a 2-month history of swelling and subsequent skin thickening in the face, trunk, and limbs and one-month history of exertional dyspnea. Physical examination revealed extensive skin thickening and fingertip scars. The modified Rodnan skin score (mRSS) was 37, indicating severe scleroderma. Nail-fold capillaroscopy revealed dilated loops, hemorrhages, and capillary loss, findings which were suggestive of SSc. Computed tomography of the chest showed ground-glass opacities and reticular changes, predominantly at posterior-basal lungs (Figure 1). A pulmonary function test revealed a reduced forced vital capacity, $64.5 \%$ of prediction. Echocardiography demonstrated preserved left ventricular systolic function and an estimated systolic pulmonary artery pressure of $35 \mathrm{~mm} \mathrm{Hg}$, without evidence of pulmonary artery hypertension. Both antinuclear and anti-Scl-70 antibodies were tested positive. Based on the 2013 EULAR-ACR criteria, diffuse SSc with ILD was diagnosed. ${ }^{10}$ In the subsequent two months, the patient received monthly 1000-mg doses of cyclophosphamide intravenously and daily $50-\mathrm{mg}$ doses of prednisolone orally, which was tapered gradually.

Two months after the first cyclophosphamide dose, he received chemomobilization with $2 \mathrm{gm} / \mathrm{m}^{2}$ of cyclophosphamide (day one) and injections of $10 \mu \mathrm{g} / \mathrm{kg} /$ day granulocyte colony-stimulating factor (days 5 to 9 ). Overall, $584.8 \times 10^{6}$ (approximately $7.9 \times 10^{6} / \mathrm{kg}$ ) CD $34^{+}$stem cells were harvested from peripheral blood and stored in liquid nitrogen for later use. No total body radiation was administered; the conditioning regimen consisted of cyclophosphamide, $50 \mathrm{mg} / \mathrm{kg} /$ day for 3 days; anti-thymocyte globulin, $2 \mathrm{mg} / \mathrm{kg} /$ day for 2 days; and cyclosporine, $3 \mathrm{mg} / \mathrm{kg} /$ day for 1 day. After conditioning, all previously harvested autologous stem cells were infused back into the patient. The infusion proceeded smoothly, and hematological recovery was rapid, with myeloid engraftment on day eight after infusion and platelet engraftment on day 11. Cyclosporine $150 \mathrm{mg} /$ day and prednisolone $20 \mathrm{mg} /$ day were prescribed for maintenance therapy and tapered gradually. Valaciclovir and trimethoprim/sulfamethoxazole (TMP/SMX) were used for one year after ASCT. Significant improvements were found in clinical, serological, and radiographic aspects (Table 1 and Figure 1). There were a decrease in the mRSS from 37 to 14 and an improvement of forced vital capacity from $64.5 \%$ to $85.73 \%$ of the predicted value. Currently, at 16 months after ASCT, the patient had no dyspnea and returned to work as a blacksmith. The cyclosporine dose was tapered to $100 \mathrm{mg} /$ day daily, and prednisolone usage was discontinued.
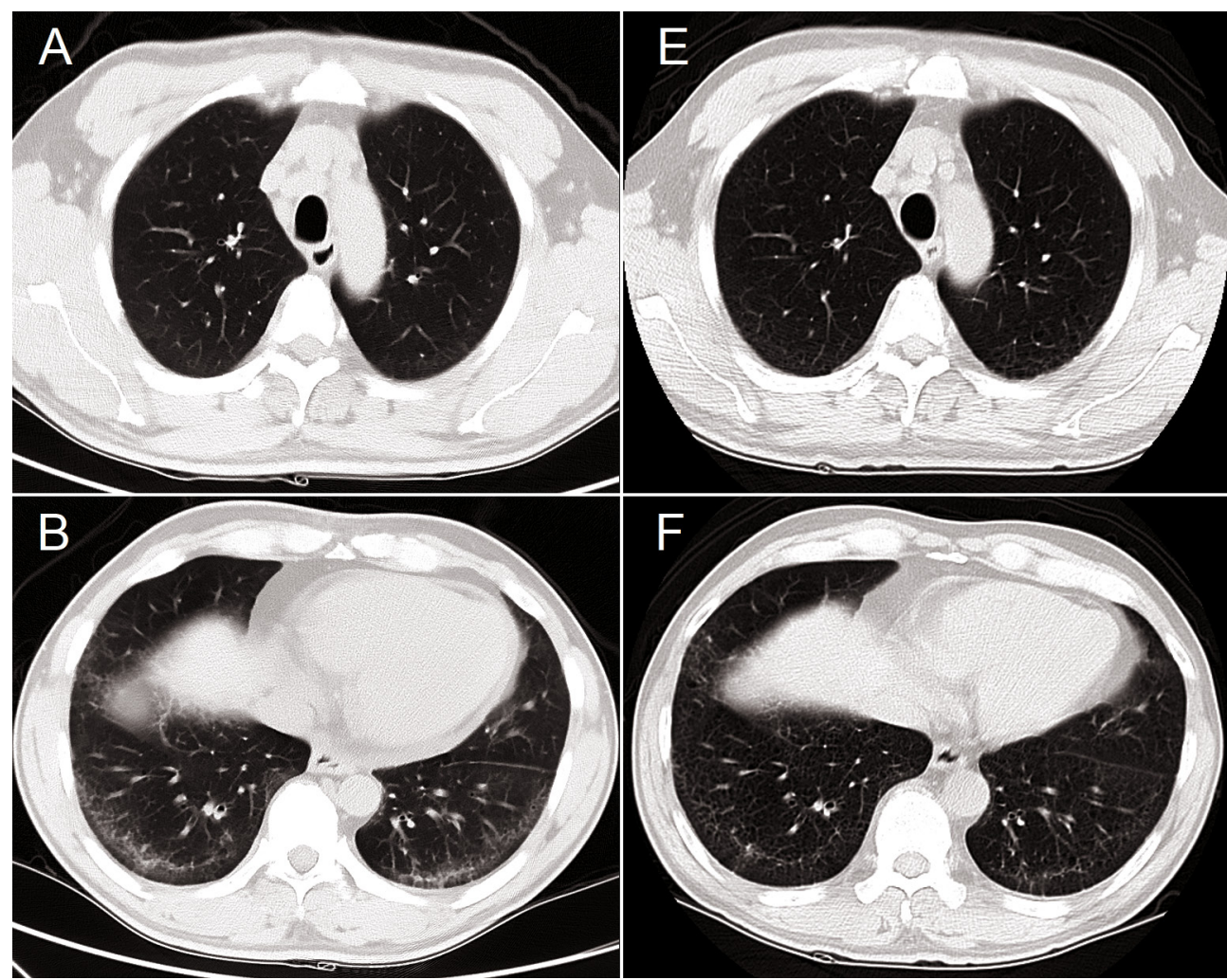

Figure 1. Representative computed tomographic (CT) images of the lungs from a patient with systemic sclerosis and interstitial lung disease (ILD). (A-D) CT images at baseline showed ground-glass opacity, traction bronchiectasis, and a reticular pattern predominantly in the basal-posterior and subpleural lung regions. (E-H) CT images 10 months after autologous hematopoietic stem cell transplantation showed partial resolution of ILD. 

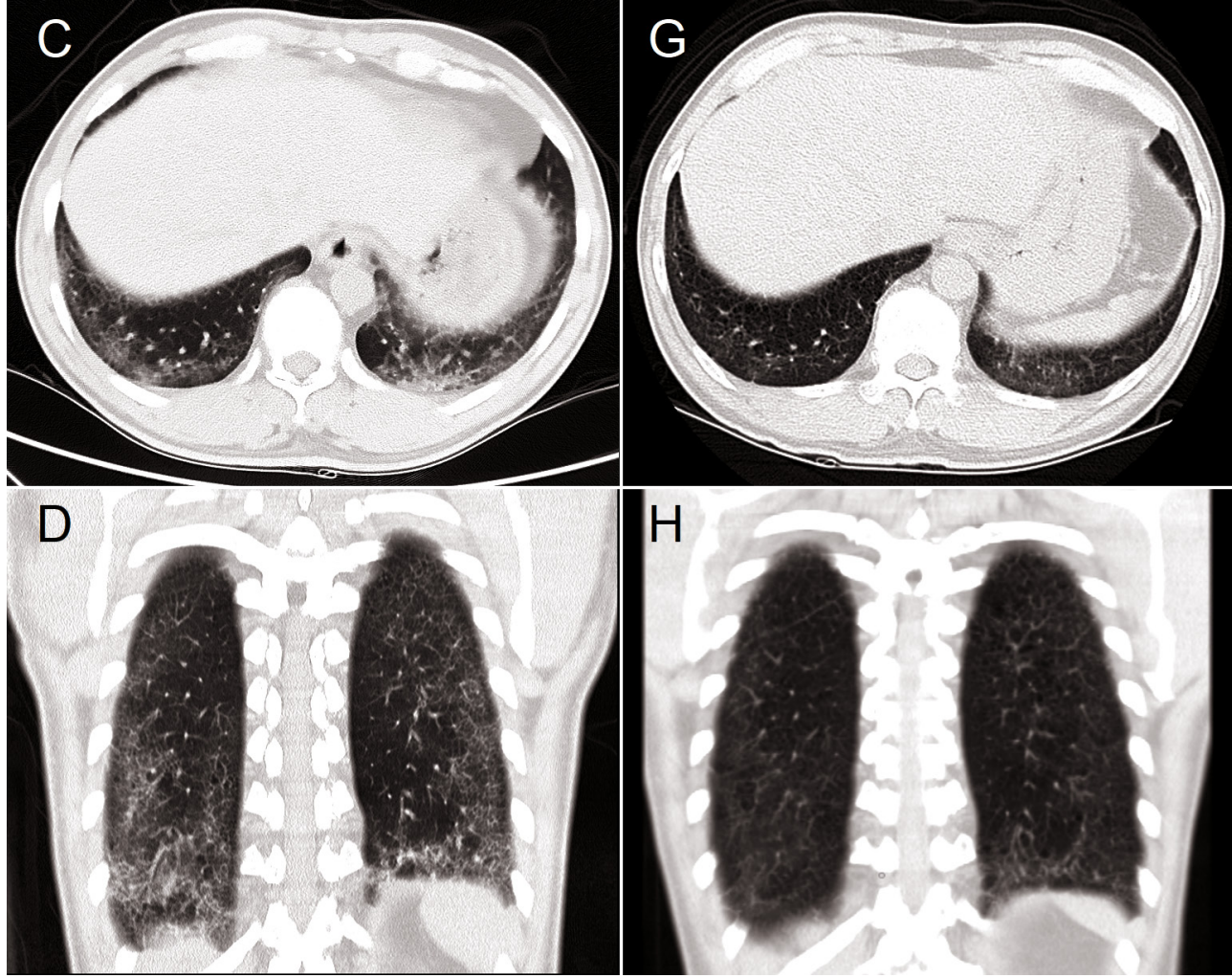

Figure 1. (Continued)

Table 1. Comparison of clinical parameters at baseline and after ASCT

\begin{tabular}{|c|c|c|c|c|}
\hline Parameters & Baseline & $\begin{array}{l}10 \text { months } \\
\text { after ASCT }\end{array}$ & $\begin{array}{l}16 \text { months } \\
\text { after ASCT }\end{array}$ & $\begin{array}{l}\text { Reference } \\
\text { range }\end{array}$ \\
\hline Serum creatinine $(\mathrm{mg} / \mathrm{dL})$ & 0.9 & 1.0 & 1.0 & $(0.7-1.3)$ \\
\hline $\operatorname{ESR}(\mathrm{mm} / \mathrm{hr})$ & 35 & 7 & 17 & $(<27)$ \\
\hline ANA titer and pattern & & & & $(<1: 40)$ \\
\hline Homogeneous & $1: 640$ & $1: 320$ & 1:160 & \\
\hline Nucleolar & $1: 320$ & $<1: 40$ & 1:40 & \\
\hline Anti-Scl-70 antibody (EliAU/mL) & 663 & 158 & 115 & $(<10)$ \\
\hline Skin fibrosis (mRSS) & 37 & 20 & 14 & NA \\
\hline \multicolumn{5}{|l|}{$\mathrm{FEV}_{1}$} \\
\hline In liters & 1.93 & 2.21 & 2.48 & \\
\hline$\%$ of predicted & 60.10 & 70.50 & 85.73 & $>80$ \\
\hline \multicolumn{5}{|l|}{ FVC } \\
\hline In liters & 2.55 & 2.69 & 3.03 & \\
\hline$\%$ of predicted & 64.50 & 69.60 & 87.23 & $>70$ \\
\hline $\mathrm{FEV}_{1} / \mathrm{FCV}(\%)$ & 75.78 & 82.09 & 81.66 & \\
\hline Six-min walk distance $(\mathrm{m})$ & 249 & 405 & 570 & $>407^{(19)}$ \\
\hline
\end{tabular}

Abbreviations: ASCT, autologous hematopoietic stem cell transplantation; ANA, antinuclear antibody; ESR, erythrocyte sedimentation rate; mRSS, modified Rodnan skin score; $\mathrm{FEV}_{1}$, forced expiratory volume in the first second; FVC, forced vital capacity; NA, not applicable. 


\section{Discussion}

Our patient had rapid progression of skin thickening and the development of symptomatic ILD early in the course of SSc; both signified a poor prognosis. He received ASCT relatively soon after disease onset. The skin and lung responses to ASCT have been satisfactory and are evident by the remarkable improvements in skin fibrosis scores, the extent of ILD, forced vital capacity, six-min walk distance, and capability for labor work.

The effects of traditional immunosuppressive therapies are limited. ${ }^{3}$ These therapies are beneficial in the short term, enabling improved lung function and skin manifestations, but have not improved long-term survival. Antifibrosis drugs, such as nintedanib, slow the progression of SSc-ILD and have some anti-inflammatory effects in animals, but not on immune dysregulation in humans with SSc. ${ }^{11}$ Alternative treatments for more advanced disease are thus needed.

Of three randomized trials of ASCT for severe SSc, all showed improvements in both the lungs and skin. ${ }^{6,7,12}$ Oneyear mortality rates related to ASCT seemed to be higher than those related to other treatments. Long-term survival was much better in patients treated with ASCT than in those treated with traditional cyclophosphamide. The American Society for Blood and Marrow Transplantation and Cellular Therapy recommends ASCT as a standard of care for severe SSc. ${ }^{13}$ Despite the recommendation, the use of ASCT for SSc in Asian countries other than Japan is seldom reported. The phase II trial in Japan showed favorable long-term survival in the ASCT arm, ${ }^{14}$ and the post hoc analysis of a phase I/II clinical trial conducted in Japan also demonstrated positive longterm safety profile of ASCT. ${ }^{8}$ Our patient exhibited satisfactory treatment responses to ASCT; moreover, no serious adverse events occurred during the follow-up period. Of importance was that our patient could return to work as a blacksmith after the ASCT treatment. This result is consistent with those reported in the clinical trials. ${ }^{15}$

The possible mechanisms of ASCT in treating autoimmune diseases include reducing the number of autoreactive $\mathrm{T}$ cells, reactivation of thymus function, renewal of T-cell receptor activity, changes in cytokine patterns, and expansion of regulatory lymphocyte populations. ${ }^{16}$ Our patient's ASCT regimen is similar to the regimen reported in ASSIST trial; ;,17 our regimen began with cyclophosphamide and anti-thymocyte globulin to induce lymphopenia and decrease the numbers of autoreactive $\mathrm{T}$ cells and autoantibodies. We expected that his immune system would recover and rebuild the immunological tolerance. Indeed, the levels of serum anti-Scl-70 antibody significantly decreased alongside the clinical responses. However, previous studies regarding the correlation of anti-Scl-70 titers with the clinical responses to ASCT have been inconclusive. The inconsistency might be attributable to differences in the enrolled patients' characteristics, conditioning regimens, or the use of total body irradiation between studies. ${ }^{6,8,13,14}$
According to the 2018 annual report of the Taiwan Society of Blood and Marrow Transplantation Registry, 4410 hematopoietic stem cell transplantations were performed between 2009 and 2018, and $44.8 \%$ of these involved autologous cells. The mortality rate at 100 days was $3.9 \%$ among patients treated with ASCT, indicating that this treatment has been extensively used in Taiwan. However, the use of ASCT for autoimmune diseases in Taiwan has not been reported. According to the Asia Pacific Blood and Marrow Transplantation Group registry, fewer than 50 patients annually received ASCT for autoimmune diseases between 2005 and $2015 .{ }^{18}$ Given the excellent survival rate after ASCT in Taiwan for hematologic disorders, ASCT might be a reasonable option for patients with autoimmune disorders, especially SSc-ILD. In addition, patient selection, accessibility, and ASCT cost as a treatment for patients with SSc-ILD should not be barriers to treatment.

In conclusion, ASCT is a beneficial treatment in patients with rapidly progressive SSc-ILD and offers better long-term survival. Since SSc-ILD is a challenging disease with a poor prognosis, we suggest that hematologists and rheumatologists collaborate in selecting patients with SSc-ILD to be treated with ASCT. This case report also demonstrates the importance of recognizing the window of opportunity for aggressive treatment in patients with rapidly progressive SSc-ILD.

\section{Ethical Approval of Studies and Informed Con- sent}

This case report was approved by the Research Ethics Committee of Hualien Tzu Chi Hospital. The authors certify that the patient has obtained an appropriate patient consent form and has given his consent for images and other clinical information to be reported. The patient understands that his name and initials will not be published, and due efforts will be made to conceal his identity, but that anonymity cannot be guaranteed.

\section{Conflicts of Interest}

The authors have no conflicts of interest relevant to this article.

\section{Author contributions}

- Yu-Jen Pan drafted and revised the manuscript.

- Wei-Han Huang reviewed the manuscript and drafted necessary corrections.

- Kuei-Ying Su reviewed and revised the manuscript, obtained patient permission, and coordinated the study.

- All authors read and approved the final manuscript.

\section{Acknowledgments}

The authors thank rheumatology nurse practitioner Tzu-Hsuan Chiu and the Tzu Chi Stem Cell Transplantation team for their medical care for the patient. 


\section{References}

1. Tyndall AJ, Bannert B, Vonk M, Airo P, Cozzi F, Carreira PE, et al. Causes and risk factors for death in systemic sclerosis: a study from the EULAR Scleroderma Trials and Research (EUSTAR) database. Ann Rheum Dis. 2010;69(10):1809-15.

2. Goh NS, Desai SR, Veeraraghavan S, Hansell DM, Copley SJ, Maher TM, et al. Interstitial lung disease in systemic sclerosis: a simple staging system. Am J Respir Crit Care Med. 2008;177(11):1248-54.

3. Volkmann ER, Tashkin DP, Sim M, Li N, Goldmuntz E, Keyes-Elstein L, et al. Short-term progression of interstitial lung disease in systemic sclerosis predicts long-term survival in two independent clinical trial cohorts. Ann Rheum Dis. 2019;78(1):122-30.

4. Tashkin DP, Elashoff R, Clements PJ, Goldin J, Roth MD, Furst DE, et al. Cyclophosphamide versus placebo in scleroderma lung disease. N Engl J Med. 2006;354(25):2655-66

5. Kowal-Bielecka O, Fransen J, Avouac J, Becker M, Kulak A, Allanore Y, et al. Update of EULAR recommendations for the treatment of systemic sclerosis. Ann Rheum Dis. 2017;76(8):1327-39.

6. Del Papa N, Pignataro F, Zaccara E, Maglione W, Minniti A. Autologous hematopoietic stem cell transplantation for treatment of systemic sclerosis. Front Immunol. 2018;9:2390.

7. van Bijnen S, de Vries-Bouwstra J, van den Ende CH, Boonstra M, Kroft L, Geurts B, et al. Predictive factors for treatment-related mortality and major adverse events after autologous haematopoietic stem cell transplantation for systemic sclerosis: results of a long-term follow-up multicentre study. Ann Rheum Dis. 2020;79(8):1084-9.

8. Ayano M, Tsukamoto H, Mitoma H, Kimoto Y, Akahoshi M, Arinobu Y, et al. CD34-selected versus unmanipulated autologous haematopoietic stem cell transplantation in the treatment of severe systemic sclerosis: a post hoc analysis of a phase I/II clinical trial conducted in Japan. Arthrit Res Ther. 2019;21(1):30.

9. Chen PM, Hsiao LT, Chen MH, Chang PM, Liu CY, Hong YC, et al. Current status of hematopoietic stem cell transplantation in Taiwan. Bone Marrow Transplant. 2008;42 Suppl 1:S133-6.

10. van den Hoogen F, Khanna D, Fransen J, Johnson SR, Baron M, Tyndall A, et al. 2013 classification criteria for systemic sclerosis: an American College of Rheumatology/European League Against Rheumatism collaborative initiative. Arthritis Rheum. 2013;65(11):2737-47.
11. Distler O, Highland KB, Gahlemann M, Azuma A, Fischer A, Mayes MD, et al. Nintedanib for systemic sclerosis-associated interstitial lung disease. N Engl J Med. 2019;380(26):2518-28.

12. Sullivan KM, Shah A, Sarantopoulos S, Furst DE. Hematopoietic stem cell transplantation for scleroderma: effective immunomodulatory therapy for patients with pulmonary involvement. Arthritis Rheumatol. 2016;68(10):2361-71.

13. Kanate AS, Majhail NS, Savani BN, Bredeson C, Champlin RE, Crawford $\mathrm{S}$, et al. Indications for hematopoietic cell transplantation and immune effector cell therapy: guidelines from the American Society for Transplantation and Cellular Therapy. Biol Blood Marrow Transplant. 2020;26(7):1247-56.

14. Nakamura H, Odani T, Yasuda S, Noguchi A, Fujieda Y, Kato M, et al Autologous haematopoietic stem cell transplantation for Japanese patients with systemic sclerosis: long-term follow-up on a phase II trial and treatment-related fatal cardiomyopathy. Mod Rheumatol. 2018;28(5): 879-84.

15. Puyade M, Maltez N, Lansiaux P, Pugnet G, Roblot P, Colmegna I, et al. Health-related quality of life in systemic sclerosis before and after autologous haematopoietic stem cell transplant-a systematic review. Rheumatology (Oxford). 2020;59(4):779-89.

16. Malmegrim KCR, Lima-Junior JR, Arruda LCM, de Azevedo JTC, de Oliveira GLV, Oliveira MC. Autologous hematopoietic stem cell transplantation for autoimmune diseases: from mechanistic insights to biomarkers. Front Immunol. 2018;9:2602.

17. Burt RK, Shah SJ, Dill K, Grant T, Gheorghiade M, Schroeder J, et al. Autologous non-myeloablative haemopoietic stem-cell transplantation compared with pulse cyclophosphamide once per month for systemic sclerosis (ASSIST): an open-label, randomised phase 2 trial. Lancet. 2011;378(9790):498-506.

18. Iida M, Kodera Y, Dodds A, Ho AYL, Nivison-Smith I, Akter MR, et al Advances in hematopoietic stem cell transplantation in the Asia-Pacific region: the second report from APBMT 2005-2015. Bone Marrow Transplant. 2019;54(12):1973-86.

19. Enright PL, Sherrill DL. Reference equations for the six-minute walk in healthy adults. Am J Respir Crit Care Med. 1998;158(5 Pt 1):1384-7. 